\title{
Comparison of Performance Efficiency of Improved Network Coding Multicast Algorithms using Data Envelopment Analysis
}

\author{
Joseph S. Soja \\ Dept. of Electrical and \\ Computer Engineering \\ Ahmadu Bello \\ University, Zaria, \\ Nigeria
}

\author{
Matthew K. Luka \\ Dept. of Electrical and \\ Electronics Engineering \\ MAUTECH, Yola, \\ Nigeria
}

\author{
Iliya T. Thuku \\ Dept. of Electrical and \\ Electronics Engineering \\ MAUTECH, Yola, \\ Nigeria
}

\author{
Saad H. Girei \\ Dept. of Computer \\ Engineering \\ Federal Polytechnic \\ Mubi, Nigeria
}

\begin{abstract}
In this paper, comparison of multicast algorithms was carried out in terms of performance Efficiency using Constant Return to Scale (CRS) and Variable Return to Scale (VRS) Model. The Improved Network Coding Algorithm (INCA) which was developed to minimize the consumption of Bandwidth during multicasting was used. The aim of the INCA is achieving cost effective multicast in order to encourage group communication over the internet. Data Envelopment Analysis was applied on the results obtained from the INCA in order to determine their performance efficiency using a number of input parameters. Simulation results obtained showed that the VRS Model performs better than the CRS Model.
\end{abstract}

\section{General Terms}

Multicasting, Modeling, Decision Making Units, Algorithms, Bandwidth Utilization, Performance Efficiency

\section{Keywords}

Performance Efficiency; Data Envelopment Analysis; Constant Return to Scale; Variable Return to Scale; Multicast Algorithm; Bandwidth Consumption

\section{INTRODUCTION}

Data Envelopment Analysis (DEA) is a linear programming technique which is used to evaluate efficiency of units which are also referred as decision making units (DMU). Multiple inputs and outputs can be included in analysis [1]. Recent research on the performance efficiency of multicast algorithms has gained significant attention especially with the deployment of Data Envelopment Analysis (DEA) as a tool for the measure of performance efficiency. A key advantage of DEA over other approaches such as the Färe, Grosskpof and Lovell approach, stochastic production frontiers, is that itmore easily accommodates both multiple inputs and multiple outputs.

Several comparisons of multicast algorithms focused largely on the effectiveness of the algorithms rather than their efficiency. DEA is an operational research tool that deals with the performance assessment of organizations or algorithms and can handle complex problems with multiple inputs and outputs [2]. Linear programming which is an optimization technique is the strength of DEA methodology. Since the work of [3], DEA has been subject to significant research publications, conferences, dissertations, and applications within the field of Engineering for measuring performance efficiency[4]. Constant return to scale and variable return to scale are the two types of DEA models usually employed for measuring the performance efficiency. Prior to its implementation in real time situation, there was a wide gap in the area of researches conducted in determining the efficiency of multicast algorithm. DEA has also been used to give new insights into activities and entities that have previously been evaluated by other methods.

Considerable amount of algorithm based-research were conducted aimed at maximizing throughput, bandwidth utilization, Evaluation of Electric Distribution utilities, energy consumption and performances [5-9]. The Improved Network Coding Algorithm (INCA) which was developed by [10] and is an extension of the research work carried out by [11].

\section{LITERATURE REVIEW}

Multicasting is used to achieve a point-to-multipoint or multipoint-to-multipoint communication. Multicasting offers scalability and improved efficiency, for applications such as audio streaming, video conferencing, online gaming and video streaming, where communication to multiple users is needed. Multicasting can either be network assisted multicast or application layer multicast. A typical implementation of network assisted multicast is Internet Protocol (IP) multicasting. An illustration of IP multicasting (IPM) and Application layer Multicasting (ALM) is provided in Fig.1 and Fig. 2 respectively.

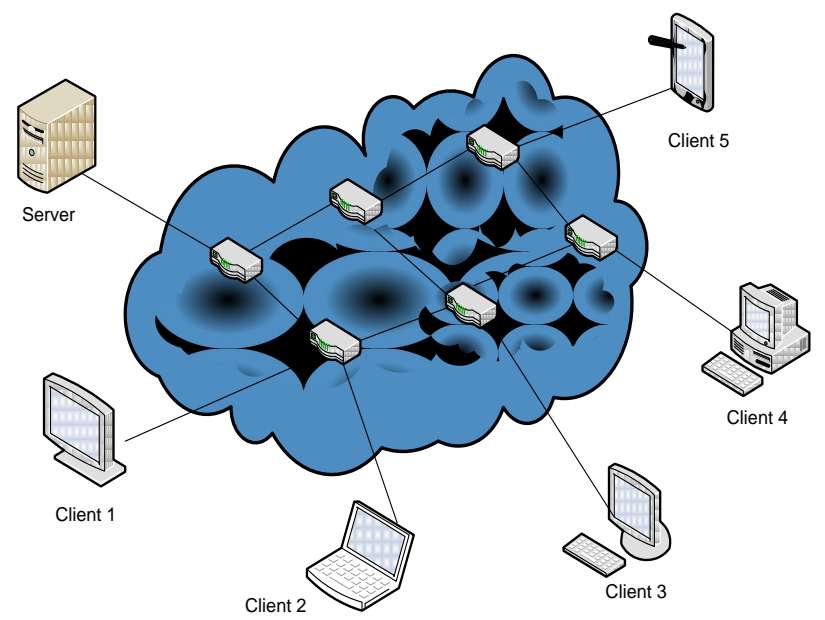

Fig. 1 IP Multicasting Architecture [12] 


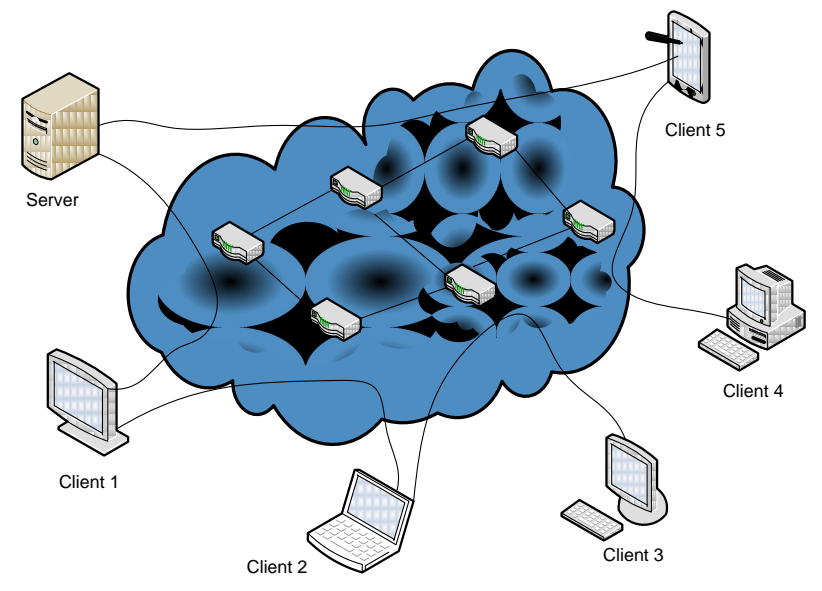

Fig. 2 Application Layer Multicasting [13]

In IPM, network routers are used to forward the message once across the network. This translates into high bandwidth and delay efficiency, lower overhead and ease of deployment. However, the user of IPM is limited by the need to deploy multi-cast capable routers across the network. IPM is also prone to flooding attacks and billing issues [12]. On other hand, ALM can be implemented as an overlay of unicast links that provides a virtual multicast network [13]. ALM overcomes the challenges of IPM but provides less efficiency in terms of bandwidth and delay. ALM is also more difficult to deploy and has higher overhead and complexity in contrast to IPM.

Cost efficiency of multicast algorithms over coded packet Network using Data Envelopment Analysis was carried out by [14]. They compared the performance of Multicast Incremental Power Algorithm (MIPA) with Network Coding Algorithm (NCA) by using Data Envelopment Analysis. Simulation showed that the NCA outperformed the Multicast Incremental Power algorithm for Cost efficient Multicast. However, the researchers were quite about the type of model employed.

Reliability target setting for electric power distribution systems was proposed in[15]. DEA was applied to determine a target of reliability indices using variable return to scale model. The aim of the research was to achieve System Average Interruption Frequency Index (SAIFI) with the help of data provided (from 2005-2008) by the Provincial Electricity Authority in Thailand (PEA). The VRS DEA model with the minimum SAIFI as the input and the average SAIFI as the output was employed.

Determination of efficient bandwidth utilization during multicast using Data Envelopment Analysis was carried out by [16] The DEA was used based on the assumption of constant return to scale model (CRS). The aim of the research is to determine the performance efficiency of the INCA with two and three parameters in terms of bandwidth utilization. Simulation results showed that the INCA with two parameters is more efficient than INCA with three parameters. However, other models such as Variable return to scale Model were not considered.

The scope of research carried out in[16]also incorporated the VRS model to determine the performance efficiency of the INCA with two and three parameters. Simulation results clearly established that the INCA with three parameters outperformed the INCA with two parameters for cost efficient
multicast.The gap identified in this review was the failure to apply the two models with a view of comparing their efficiency. There is need to apply the two models on the same scenario so as to determine which of the model is more efficient and hence the motivations of this research work.

Performance efficiency using Data Envelopment Analysis employ the use of two models namely, Variable Return to Scale called BCC Model (Banker, Charnes and Cooper) and Constant Return to Scale called CCR model (Charnes, Cooper, and Rhodes). The two models have been separately used to determine the performance efficiency of multicast algorithms over wireless network. In this paper, the efficiencies of the two models will be compared on the simulation results obtained for the Improved Network Coding Algorithm with two and three parameters. Recent researches conducted by [16] -[18] focused on performance efficiency of the INCA using VRS and CRS models. The INCA can be expressed as linear programming optimization problem of equation (1) [6]:

$$
\min (q) \sum_{(i, j)}^{n} C_{i j} y_{i j} \quad \forall(i, j) \in V, \quad i \neq j
$$

Subject to:

$$
\begin{gathered}
C_{i j} \leq C_{i, a_{j}^{i}} \quad \forall(i, j) \in A, \quad a_{j}^{i} \neq j \\
\delta(p) \leq 400 \quad \forall(i, j) \in V, \quad i \neq j \\
\rho(l) \leq 4 \quad i \neq j, \quad \forall(i, j) \in V \\
\sum_{y=1}^{n} y_{i j}>0 i \neq j, \quad \forall(i, j) \epsilon \frac{v}{\{s\}}
\end{gathered}
$$

Where $\mathrm{C}_{\mathrm{ij}}$ is the cost of bandwidth used during multicast $\operatorname{andy}_{\mathrm{ij}}$ is distance from one node to the other. $\left(\mathrm{i}, \mathrm{a}_{\mathrm{j}}^{\mathrm{i}}\right)$ is the arc originating in node $\mathrm{i}$ with the lowest cost, $\mathrm{q}$ is the objective function to be minimized, $\mathrm{p}$ is the packet delay, $\mathrm{l}$ is the packet loss, $\mathrm{i}$ denotes the source node and $\mathrm{j}$ represents the destination node.

The aim of this paper is to compare the performance efficiencies of the two multicast algorithms namely; the Improved Network Coding Algorithm (INCA) with two and three Parameters using both the CRS/VRS models with the aim of establishing which of the models is more efficient during multicasting over wireless network.

\section{DEA MATHEMATICAL MODEL}

DEA is a "data-oriented" approach for evaluating the performances of a set of entities called Decision Making Units (DMUs). DMUs convert multiple inputs into multiple outputs. It allows researchers to compare relative efficiency of a production unit by determining the efficient production unit as benchmark using either constant Return to scale or variable return to scale model [17]. It has been applied in evaluating the performances of different classes of entities engaged in a number of activities in different contexts.

There are a number of DEA models. The main focus of this research is to compare the performance efficiency of the CCR-model and the BCC model. The main difference between the two models is the treatment of returns-to- scale. BCC allows for variable returns- to-scale; CCR assumes that each DMU operates with constant returns- to- scale. 


\subsection{Constant Return to Scale Model}

Constant return to scale model introduced a measure of efficiency for each DMU that is obtained as a maximum of a ratio of weighted outputs to weighted inputs [18].

$$
\min \left(h_{0}\right)=\theta_{0}
$$

Subject to:

$$
\begin{gathered}
\sum_{J=1}^{n} \lambda_{j} y_{r J} \geq y_{r 0} \quad r=1,2, \ldots S \\
\theta_{0} x_{i 0}-\sum_{J=1}^{n} \lambda_{j} y_{r J} \geq y_{r 0} \\
\lambda_{j} \geq 0 \quad j=1,2, \ldots n
\end{gathered}
$$

$\mathrm{y}_{\mathrm{rJ}}$ is the amount of output, $\mathrm{r}$, produced by DMU, $\lambda_{\mathrm{j}}$ is the weight of input $\mathrm{j}$ and $\mathrm{x}_{\mathrm{i} 0}$ is the amount of input $\mathrm{j}$ utilized by DMU q.

The constraints of equation (1) and equation (7) showed the mathematical model for Constant Return to Scale Model. Both linear problems yield the optimal solution $\theta_{*}$, which is the efficiency score (constant Return to scale -efficiency) for the particular $D M U_{0}$

\subsection{Variable Return to Scale Model}

Variable-returns-to- scale can be realized by adding convexity condition for $\lambda_{j}$. The addition of convexity condition in Equation (10) to the CRS DEA model is called the Variable return to scale Model.

$$
\sum_{j=1}^{n} \lambda_{j}=1
$$

The input-oriented VRS-model for the $D M U_{0}$ can be written formally as the CRS model subject to the constraints of equation (7) - (10). By solving the model for each DMU, the VRS -efficiency scores are obtained.

\section{SIMULATION RESULTS AND DISCUSSION}

Fig. 3 shows the result of simulations for the INC algorithm using two parameters (packet delay and cost of bandwidth). The result indicates an increase in bandwidth consumption with increase in the number of multicasting participants. It can also be seen that the cost is better when more routing nodes are available. This is due to fact that more routing nodes provide more alternative communication routes that translates into a more cost effective multicasting. The cost of bandwidth is further improved by using an additional parameter (packet loss) as shown in Fig. 4. In order to achieve better cost efficiency, The VRS and CRS models were applied to determine the number DMUs in an ensample of 20, 50 and 60 routing nodes. The results shown in Fig. 5 - Fig. 7, indicates that the performance efficiency of both the VRS and CRS model improve with increase in the ensample of DMUs. However, the performance of the VRS is seen to be more consistent than that of the CRS model, which tends to deteriorate with increase in the number of participants.

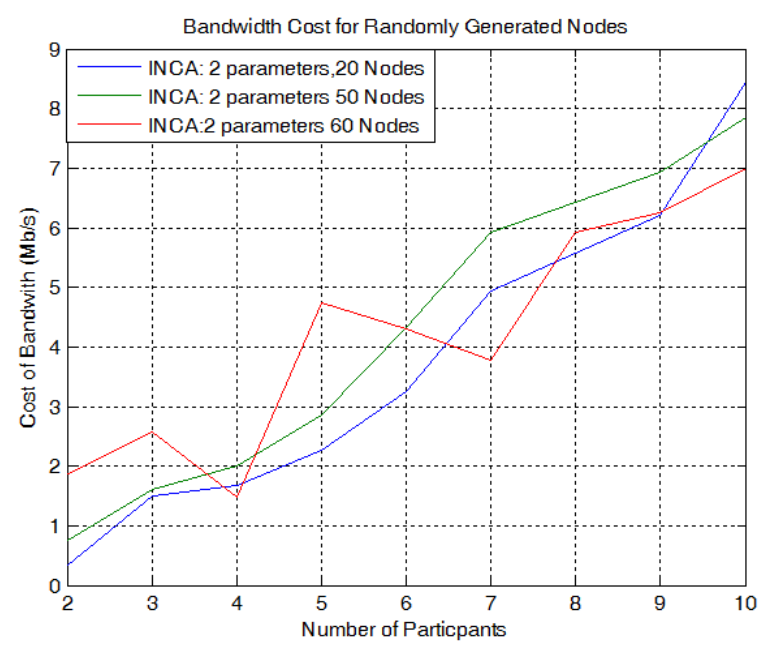

Fig. 3 Cost of Bandwidth Consumption for INCA with Two Parameters

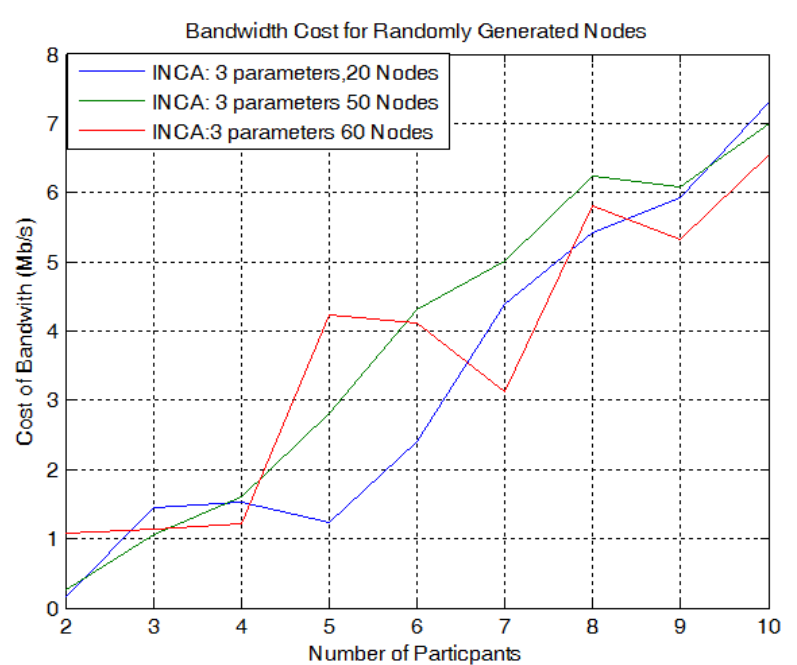

Fig.4 Cost of Bandwidth Consumption for INCA with Three Parameters

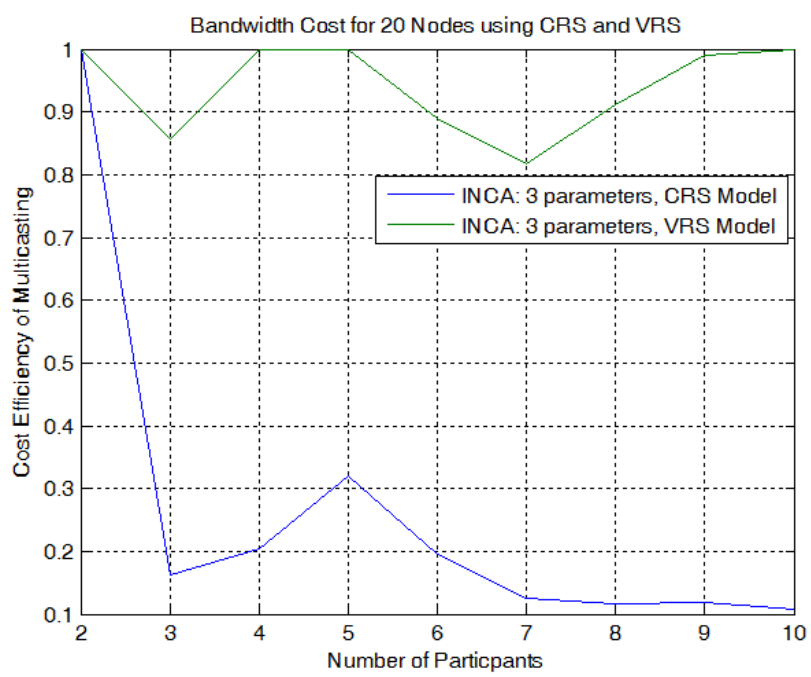

Fig.6 Performance Efficiency of INCA with Three Parameters using CRS and VRS Models for 20 Nodes 


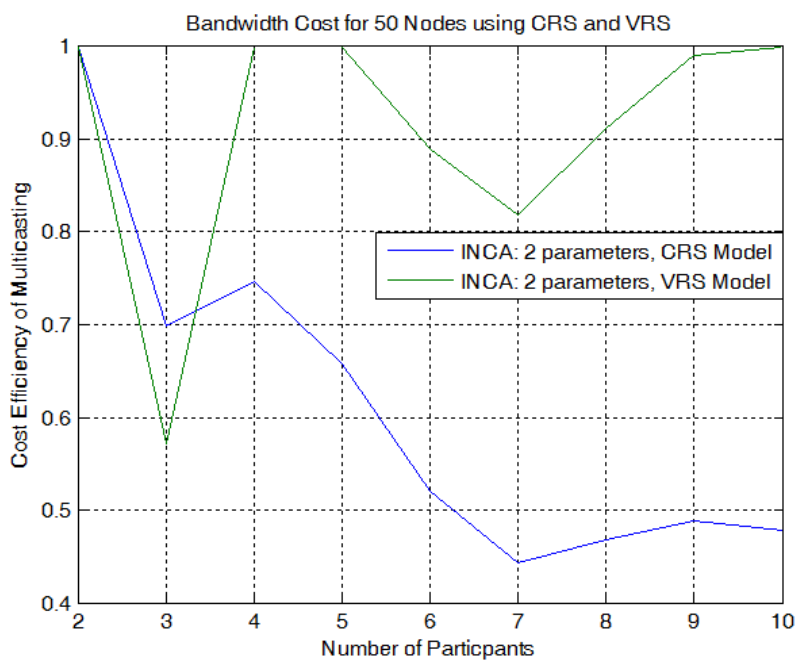

Fig. 5 Performance Efficiency of INCA with three Parameters using CRS and VRS Models for 50 Nodes

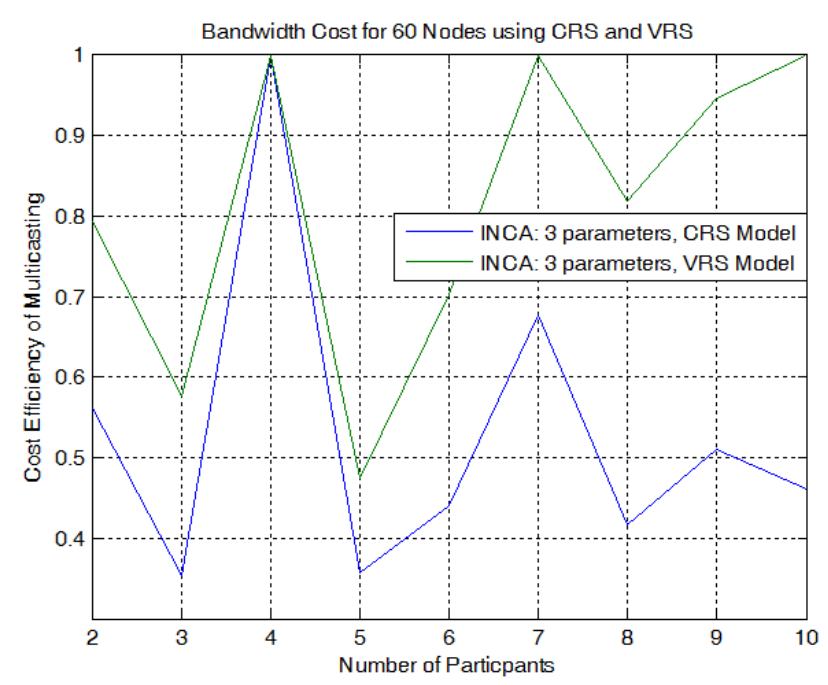

Fig. 7 Performance Efficiency of INCA with Three Parameters using CRS and VRS Models for 50 Nodes

\section{CONCLUSION}

In this work, comparison of performance efficiency of Improved Network Coding algorithm using the VRS and CRS Data Envelopment Analysis models were carried out. The VRS DEA model is similar to the CRS model; the only difference being a convexity constraint on the weight of the input. The VRS model was observed to be relatively consistent in the face of increasing number of participants when an ensample of Decision Making Units (routing Nodes) is used. This is a superior performance to the CRS model, which is characterized by a decrease in performance efficiency under the same network conditions and ensample of DMUs. Further work can be done to consider the technical efficiency of the INCA with two and three parameters.

\section{REFERENCES}

[1] Liang Liang, Feng Yang, and W. D. C. a. J. Zhu, "DEA models for supply chain efficiency evaluation," Annals of Operations Research, vol. 145, pp. pp 35-49, 2006.
[2] C. R. Subshash, "Data Envelopment Analysis: T Practices and Techniques of Economics and Operational Research.," Cambridge University Press, 2004.

[3] A. Charnes, W. W. Cooper, and E. L. and Rhodes, "Measuring the efficiency of decision making units," European Journal of Operational Research, vol. 2, pp. 429-444, 1978.

[4] D. K. J. a. R. Shrestha, "Measuring Efficiency of Hydropower Plants in Nepal Using Data Envelopment Analysis," IEEE Trans. on Power Systems, vol. vol. 21, 2006.

[5] A. A. Ajibesin, G. M. Wajiga, M. R. Odekunle, and O. K. Egunsola, "Energy-efficient Multicast in wired and wireless Networks: Analysis and performance Measures," Computer science and artificail intellegent laboratory, vol. 65, pp. 23-29, 2013.

[6] S. J. Soja, S. Muhammed Sani, A. M. S Tekanyi, and S. Garba, "Improvement of Multicast Network Coding Algorithm for Bandwidth Utilization over Coded Packet Wireless Network," International Engineering conference, Minna pp. 256-272, 2015.

[7] B. T. Swapna, A. Eryilmaz, and N. B. Shroff, "Throughput-delay analysis of random linear network coding for wireless broadcasting," Information Theory, IEEE Transactions on, vol. 59, pp. 6328-6341, 2013.

[8] H. Venkataraman, A. d'Ussel, T. Corre, C. H. Muntean, and G.-M. Muntean, "Performance analysis of real-time multimedia transmission in $802.11 \mathrm{p}$ based multihop hybrid vehicular networks," in Proceedings of the 6th International Wireless Communications and Mobile Computing Conference, 2010, pp. 1151-1155.

[9] Anil Pahwa, Xiaoming Feng, and D. Lubkeman., "Performance Evaluation of Electric Distribution Utilities Based on Data Envelopment Analysis," Ieee Transactions On Power Systems, vol. 17, pp. 400-4005, 2002.

[10] Soja .J. S, S. M. sani, S. Garba, and A. M. S. Tekanyi, "Development of an Improved Multicast Algorithm for Interpreting the Cost of Bandwidth within the Channel during Multicast using Shannon-Hartley Channel Capacity Theorem," International Journal of Engineering Research \& Technology (IJERT), vol. 4, pp. 252-255, 2015.

[11] A. A. Ajibesin, N. Ventura, H. A. Chan, A. Murgu, and O. K. Egunsola, "Performance of Multicast Algorithms Over Coded Packet Wireless Networks," in Computer Modelling and Simulation (UKSim), 2012 UKSim 14th International Conference on, 2012, pp. 596-600.

[12] C. Diot, B.N Levine, B. Lyles, H. Kassem, and D. Balensiefen, "Deployment Issues for the IP Multicast service and Architecture," "IEEE Network Mag, vol. 14 , pp. 78-88, 2000.

[13] M. Hosseini, D. Tanvir, S. Shirmohammadi, and N. D. Georgannas., "A Survey of Application Layer Multicast protocols," ed, 2001.

[14] A. A. Ajibesin, N. Ventura, A. Murgu, and H. A. Chan, "Cost-efficient multicast over coded packet wireless networks using data envelopment analysis," in Consumer 
Communications and Networking Conference (CCNC), 2013 IEEE, 2013, pp. 546-551.

[15] J. K. a. P. Raphisak, "A Data-Envelopment-Analysis Inspired Method for Reliability Target Setting," Annals of Operations Research,, vol. 34, pp. 1-4, 2008.

[16] Joseph Stephen Soja, S. M. Sani, and A. M. SunkaryTekanyi, "Determination of Efficient Bandwidth Utilization using Data Envelopment Analysis,"
International Journal of Computer Applications, p. in press, 2016.

[17] R. D. Banker, W. W. Cooper, L. M. Seiford, R. M. Thrall, and J. Zhu, "Returns to scale in different DEA models," European Journal of Operational Research, vol. 154, pp. 345-362, 2004.

[18] Igor Jemric and B. Vujcic, "Efficiency of Banks in Croatia: A DEA Approach," presented at the Comparative Economic Studies, Xliv, 2002. 